\title{
Efeito do extrato da casca de Syzygium cumini sobre a atividade da acetilcolinesterase em ratos normais e diabéticos
}

\author{
Syzygium cumini bark extract effect on acetylcholinesterase activity in normal and diabetic rats
}

\author{
Cinthia Melazzo Mazzanti ${ }^{1}$ Deila Rosély Schossler ${ }^{2}$ Andreane Filappi ${ }^{3}$ Danívia Prestes ${ }^{3}$ \\ Adriane Cismoski Silva ${ }^{4}$ Maisa Correa ${ }^{5}$ Maria Rosa Chitolina Schetinger ${ }^{6}$ Vera Maria Morsch ${ }^{7}$ \\ Gilberto Lunkes $^{8}$ Wellington de Abreu Gonzaga ${ }^{9}$ Marcelo Cecim ${ }^{10}$
}

\section{RESUMO}

Este estudo verificou a eficiência do extrato etanólico da casca de Syzygium cumini sobre o sistema colinérgico de ratos normais e diabéticos induzidos com aloxano. Os animais foram divididos em grupo controle $(C)$, tratado com Syzygium cumini (TS), diabético $(D)$ e diabético tratado com Syzygium cumini (DS). A atividade da acetilcolinesterase (AChE) foi analisada nas seguintes estruturas cerebrais: cerebelo, córtex, estriado e hipocampo. O extrato etanólico da casca de Syzygium cumini na dose de $1 \mathrm{~g}^{\mathrm{kg}} \mathrm{g}^{-1}$ foi administrado diariamente por um período de trinta dias. Foi verificado após este período que o extrato inibiu a atividade da AChE no cerebelo e córtex cerebral dos ratos do grupo DS $(P<0,05)$, comparado com o TS. No estriado houve um aumento significativo na atividade da AChE nos ratos do grupo $T S(P<0,01)$ comparado com o $C$, e no hipocampo não foi encontrada nenhuma variação significativa. Esses resultados indicam que o extrato da casca do Jambolão, possui um efeito inibitório da AChE no cerebelo e córtex cerebral e um efeito ativador sobre essa enzima no estriado, indicando uma possível alteração na funcionalidade do sistema colinérgico nestas estruturas cerebrais.

Palavras-chave: casca, diabetes, sistema colinérgico, Syzygium cumini.

\section{ABSTRACT}

The present study verified the efficiency of the bark ethanol extract of Syzygium cumini on the cholinergic system of normal and alloxan induced diabetic rats. Thirty-nine female rats were divided in control $(C)$, treated with Syzygium cumini (TS), diabetic (D) and diabetic treated with Syzygium cumini (DS). The activity of acetylcholinesterase (AChE) was analyzed in the following cerebral structures: cerebellum, cortex, striatum and hippocampus. The extract of the bark of Syzygium cumini in the dose of $1 \mathrm{~g} . \mathrm{kg}^{-1}$ was administered orally daily for a period of thirty days. After this period the extract inhibited the activity of the AChE in the cerebellum and cerebral cortex of the rats in the DS group $(P<0.05)$ as, compared to TS. In the striatum there was a significant increase in the activity of the AChE in rats of the TS group $(P<0.01)$ when compared to the $C$ group, and in the hippocampus there was no significant variation. These results indicate that the bark extract of "Jambolão" has an inhibitory effect on AChE in the cerebellum and cerebral cortex and an stimulatory effect on striatum, indicating a possible alteration in the functionality of the cholinergic system in such cerebral structures.

Key words: bark, cholinergic system, diabetes, Syzygium cumini.

\section{INTRODUÇÃO}

A terapia com plantas medicinais vem sendo muito utilizada em vários países do mundo como um tratamento alternativo para o diabetes melito. Alguns aspectos têm despertado o interesse científico dos pesquisadores, principalmente estudos sobre o valor

${ }^{1}$ Médico Veterinário, Doutorando de Bioquímica da Universidade Federal do Rio Grande do Sul (UFRGS).

${ }^{2}$ Médico Veterinário. Doutor Professor Adjunto, Departamento de Morfologia da Universidade Federal de Santa Maria (UFSM).

${ }^{3}$ Médico Veterinário. Esp. Aluna do Programa de Pós-graduação em Medicina Veterinária da UFSM.

${ }^{4}$ Farmacêutico, Aluno de Pós-graduação em Bioquímica Toxicológica da UFSM.

${ }^{5}$ Acadêmico do Curso de Farmácia da UFSM. Bolsista de Iniciação Científica-CNPq/UFSM.

${ }^{6}$ Biólogo, Professor Adjunto, Departamento de Química da UFSM.

${ }^{7}$ Químico, Professor Adjunto, Departamento de Química da UFSM

${ }^{8}$ Farmacêutico. MSc. UFSM.

${ }^{9}$ Químico, Doutorando do Curso de Química da UFSM.

${ }^{10}$ Médico Veterinário. PhD. Prof. Adjunto. Departamento de Clínica de Grandes Animais da UFSM, 97105-900, Santa Maria, RS. E-mail: mcecim@hcv.ufsm.br. Autor para correspondência. 
terapêutico, risco e toxicidade dessas plantas correntemente empregadas, não apenas no diabetes, mas em um grande número de doenças (BRAGANÇA, 1996).

Syzygium cumini (L.) skeels é uma das plantas mais utilizadas no tratamento do diabetes melito. É uma árvore originária da Índia, pertencente à família das Mirtáceas, vulgarmente conhecida no Brasil como Jambolão (BRAGANÇA, 1996). O efeito dessa planta sobre os níveis de glicose sanguínea tem sido avaliado em diversos estudos. Os resultados encontrados por SOARES et al. (2000) e GROVER et al. (2000) que utilizaram respectivamente a folha e a semente do Jambolão demonstraram ação hipoglicemiante da planta. PRINCE et al. (1998), além de comprovarem o efeito hipoglicemiante da semente de jambolão, também verificaram que a planta possui um efeito antioxidante.

Além de sua atividade antimicrobiana, o decocto da casca é usado para o tratamento de diarréias e desordens gastrintestinais (BRAGANÇA, 1996). Por outro lado, Syzygium cumini demonstrou ação sedativa, anticonvulsivante (LIMA et al., 1998) e depressora do sistema nervoso central (SNC) (CHAKRABORTY et al., 1986).

A diabetes melito está associada com alterações cognitivas, estruturais e fisiológicas do cérebro, condição esta denominada encefalopatia diabética. Estudos recentes descreveram os sintomas nervosos da diabetes como degeneração cerebral acelerada, condição em que o estado diabético altera a plasticidade sináptica modificando o mecanismo de regulação da homeostase celular, com consequente disfunção na atividade dos neurotransmissores na fenda sináptica (BIESSELS et al., 2002).

A acetilcolina ( $\mathrm{ACh}$ ) é um dos neurotransmissores excitatórios mais estudados no SNC e no sistema nervoso periférico (SNP), sendo sintetizada a partir de acetil coenzima A (acetil CoA), formado durante a respiração celular e da colina, um importante produto do metabolismo dos lipídeos (TAYLOR \& BROWN, 1994). É importante nas funções desempenhadas pelo sistema nervoso central e tem sido associada com as funções cognitivas, processamento de informações sensoriais, organização cortical do movimento e controle do fluxo sanguíneo cerebral (SCREMIN et al., 1997).

A acetilcolinesterase (AChE) é uma serina hidrolase que desempenha um papel essencial no mecanismo colinérgico, catalisando a hidrólise do substrato ACh em ácido acético e colina (MARCEL et al., 1998). A AChE é encontrada, principalmente nas sinapses do SNC, SNP parassimpático e junção neuromuscular. É uma importante enzima regulatória que controla a transmissão de impulsos nervosos através de sinapse colinérgica pela hidrólise do neurotransmissor excitatório ACh, terminando portanto, sua ação (MILATOVIC \& DETTBARN, 1996; MULLER et al., 2002). Considerando que Syzygium cumini possui um efeito depressor no SNC, torna-se relevante o estudo da atividade da $\mathrm{AChE} \mathrm{em}$ diferentes estruturas cerebrais (cerebelo, córtex cerebral, estriado e hipocampo), uma vez que na literatura disponível, não foram encontrados dados referentes à atividade dessa enzima, relacionada com a planta em estudo.

Dessa forma, este trabalho foi conduzido para investigar o efeito do extrato da casca de Syzygium cumini na atividade da enzima AChE em ratos normais e diabéticos induzidos com aloxano.

\section{MATERIAL E MÉTODOS}

O estudo foi realizado em 39 ratas adultas da linhagem Wistar, pesando entre 220 e $300 \mathrm{~g}$, provenientes do Biotério Central da Universidade Federal de Santa Maria (UFSM). A dieta sólida ${ }^{a}$, bem como a hídrica, foram fornecidas ad libitum. Antes do início do experimento, os animais passaram por um período de adaptação de dez dias.

As cascas do caule de Syzygium cumini foram coletadas em maio de 2001, no município de Santa Maria (Rio Grande do Sul, Brasil). A identificação da planta foi realizada pelo biólogo Gilberto Zanetti (inscrito no "Cadastro Técnico Federal de Atividades e Instrumentos de Defesa Ambiental" com o número 4131/98, conforme exigência do IBAMA) e então registrada no Herbário de Plantas Medicinais e Tóxicas do Departamento de Farmácia Industrial da UFSM, pela exsicata de número 160. O material (casca do caule) de Syzygium cumini foi seco, inicialmente sob luz, evitando-se a presença de fungos e, após em uma estufa, à temperatura de $50^{\circ} \mathrm{C}$, sendo em seguida processado em moinho Wiley. De posse do material moído, passou-se a fazer a extração a frio, utilizando-se etanol (Álcool etílico $96^{\circ} \mathrm{GL}$ ) como solvente, e depois de várias extrações e com a evaporação do solvente em rotavapor com pressão reduzida, obteve-se um resíduo escuro viscoso, denominado extrato bruto com rendimento de $10 \%$ do peso $(4100 \mathrm{~g})$ da casca do caule.

Para indução do diabetes melito insulino dependente (DMID), administrou-se, aloxano ${ }^{\mathrm{b}}$ (diluído a $2 \%$ em solução de citrato de sódio $0,05 \mathrm{M}, \mathrm{pH} 4,5$ ) na dose de $150 \mathrm{mg} / \mathrm{kg}$, após um período de jejum de 24 horas, via intraperitoneal, dose única. Após 6 horas 
da indução, foi fornecida glicose $10 \%$ como única fonte hídrica, durante 24 horas para evitar uma hipoglicemia fatal, devido à liberação maciça de insulina que ocorre após destruição das células ß pelo aloxano. Após 15 dias, a glicose sanguínea foi determinada, durante o período matinal, com os animais em jejum de 12 horas. Os ratos que apresentaram uma glicemia inferior a $180 \mathrm{mg} \mathrm{dL}^{-1}$, foram novamente induzidos, seguindo o mesmo protocolo. Somente os animais com glicemia de jejum superior a $180 \mathrm{mg} \mathrm{dL}^{-1}$ foram considerados diabéticos e selecionados para o experimento.

Os animais foram divididos, de forma aleatória em 4 grupos: Grupo1: controle $(\mathrm{C}=11)$; Grupo 2: Tratado com Syzygium cumini (TS=9); Grupo 3: Diabético ( $\mathrm{D}=10)$; Grupo 4: Diabético tratado com Syzygium cumini (DS=9). Esses 4 grupos mimetizam situações de normalidade, prevenção, enfermidade e tratamento respectivamente. Os grupos 1 (C) e 3 (D) receberam diariamente $2 \mathrm{~mL}$ de água destilada através de uma sonda oroesofágica, e os grupos 2 (TS) e 4 (DS) receberam diariamente o extrato da casca de Syzygium cumini na dose de 1g/ $\mathrm{kg}$ de peso vivo. O tratamento foi realizado por um período de 30 dias e após esse período, os animais foram submetidos à eutanásia sob anestesia inalatória em campânula com éter. Logo após, a cabeça foi retirada e cuidadosamente aberta, expondo o encéfalo, que foi colocado sobre um papel filtro umedecido com Meio I (Sacarose 0,32 M, Tris 5,0mM, EDTA 0,1 mM, pH 7,5). Em seguida, as estruturas cerebrais (cerebelo, córtex cerebral, estriado e hipocampo) foram separadas, homogeneizadas isoladamente e a atividade da AChE foi determinada pelo método de ELLMAN et al. (1961), modificado por ROCHA et al. (1993) em cada uma das estruturas cerebrais.

Realizou-se análise estatística dos valores da atividade da AChE nas estruturas (cerebelo, córtex cerebral, estriado e hipocampo), entre os diferentes grupos. Aplicou-se uma análise de variância (ANOVA) de uma via, seguido do teste de comparações múltiplas de Tukey-Kramer. Os resultados são expressos por média \pm erro padrão da média.

\section{RESULTADOS E DISCUSSÃO}

Neste estudo foi encontrada uma diminuição significativa na atividade da AChE no cerebelo dos ratos do grupo DS $(\mathrm{P}<0,05)$, comparado com o C, TS e D (Figura 1A). Observa-se com esse resultado que se associando o extrato do Jambolão e a diabetes induzida pelo aloxano, os animais apresentaram inibição da enzima. Observou-se também uma diminuição significativa na atividade da AChE no córtex cerebral de ratos DS $(\mathrm{P}<0,05)$, comparado com o grupo TS (Figura 1B).

Esses resultados indicam que o extrato da casca de Syzygium cumini na dose de $1 \mathrm{~g} \mathrm{~kg}^{-1}$, de peso vivo, demonstrou um efeito inibitório sobre a atividade da acetilcolinesterase, sendo esta responsável pela degradação do neurotransmissor acetilcolina (ACh), que é co-lançado na fenda sináptica (TAYLOR \& BROWN,1994; BIESSELS et al., 2002), o que leva a supor que o uso farmacológico deste extrato pode interferir com a funcionalidade do SNC. Além disso, sugere-se que o estado diabético induzido pelo aloxano (PRINCE et al., 1998; GROVER et al., 2000; SOARES et al., 2000), associado à terapia com o extrato (BRAGANÇA, 1996), intensificaram a diminuição da AChE.

No estriado, estrutura rica em vias colinérgicas (MILATOVIC \& DETTBARN, 1996; SCREMIN et al., 1997; MARCEL et al., 1998; MULLER et al., 2002)), observou-se que o grupo TS $(\mathrm{P}<0,05)$ apresentou atividade enzimática aumentada quando em comparação com os grupos C, D e DS (Figura 1C). Isto sugere que o tratamento com o extrato da planta por si só foi capaz de intensificar a atividade da AChE nesta estrutura, o que merece estudos mais aprofundados. No entanto, no hipocampo não foi encontrada nenhuma variação significativa na atividade da enzima AChE nos grupos estudados (Figura 1D).

A partir dos dados obtidos, observou-se que a indução do diabetes com aloxano, por si só, não alterou a atividade da enzima $\mathrm{AChE}$ enquanto que o tratamento com o extrato de Syzygium cumini foi capaz de fazê-lo. Em contrapartida, LAKHMAN \& KAUR (1994) e KHANDKAR et al. (1995) observaram um aumento na atividade da AChE após a indução com o aloxano, concluindo que a atividade da AChE no cérebro é afetada diretamente pelo nível de glicose sanguínea.

Em um estudo realizado por CHAKRABORTY et al. (1986), a semente do jambolão produziu alterações comportamentais, redução na temperatura corporal, ação analgésica, ação depressora do SNC e nenhuma atividade anticonvulsivante. Também foi relatado que vários flavonóides encontrados na casca dessa planta (BRAGANÇA, 1996) possuem atividade ansiolítica e/ou sedativa, parcialmente atribuída a sua afinidade para o receptor benzodiazepínico central (LIMA et al., 1998).

Os efeitos inibitórios indicados neste estudo sobre a atividade da AChE, no cerebelo e 


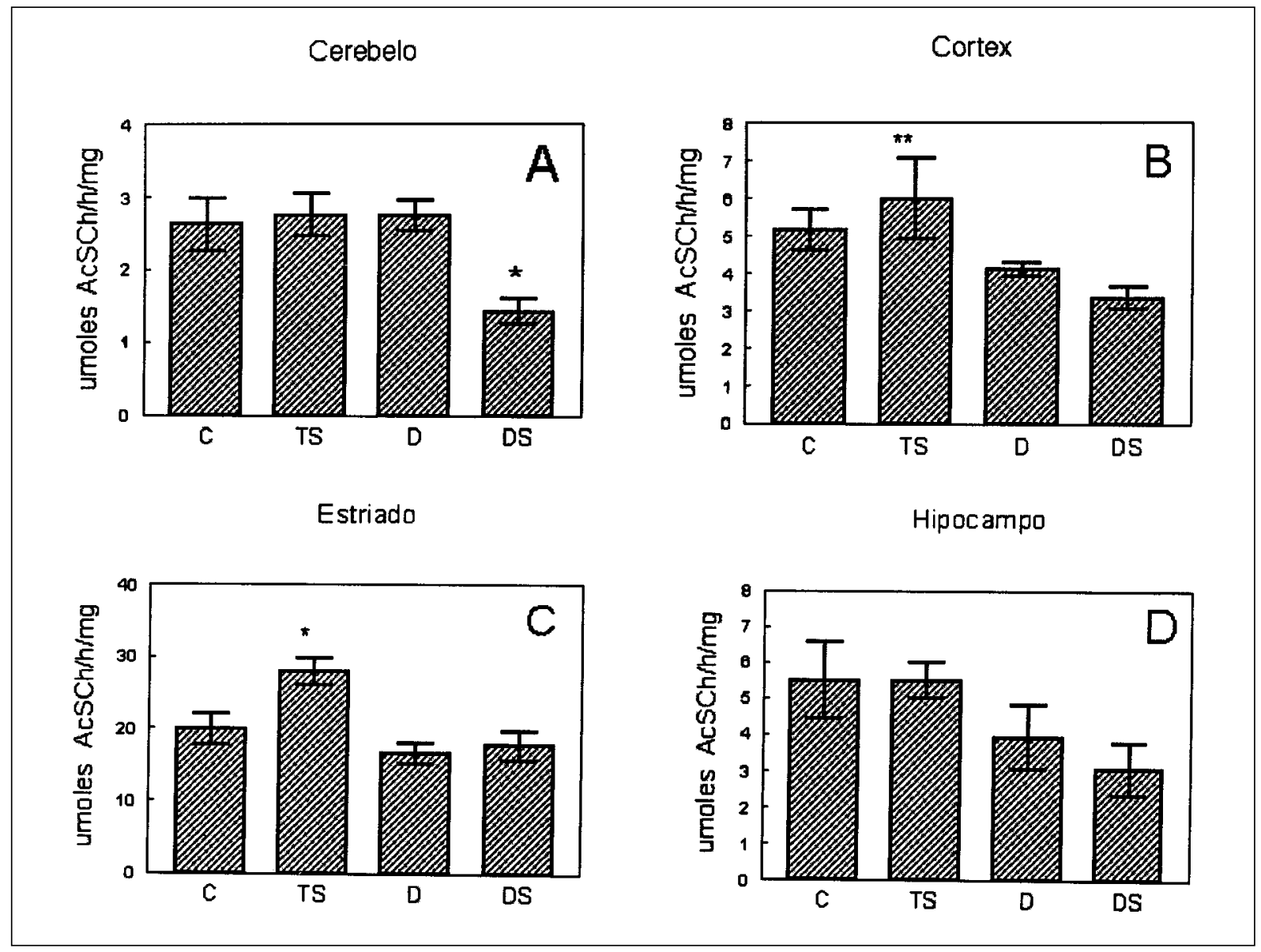

Figura 1 - Atividade da acetilcolinesterase (AChE) no cerebelo, córtex cerebral, estriado e hipocampo de ratos normais e diabéticos tratados com o extrato da casca de Syzygium cumini. C= Controle; TS= Tratado com Syzygium cumini; D=Diabético; DS= Diabético tratado com Syzygium cumini.

* $(\mathrm{P}<0,05)$ em relação aos demais

${ }_{* *}(\mathrm{P}<0,05)$ DS em relação ao TS

Os valores representam média $\pm \mathrm{EPM}$ ( $\mathrm{n}=9$ a 11 animais).

$\mathrm{EPM}=$ erro padrão da média.

córtex cerebral podem estar associados aos componentes químicos contidos na casca do jambolão agindo nos receptores colinérgicos, sugerindo assim uma alteração no sistema nervoso central.

\section{CONCLUSÕES}

Com base nos resultados obtidos neste estudo, pode-se concluir que o extrato da casca de Syzygium cumini na dose de $1 \mathrm{~g} \mathrm{~kg}^{-1}$ possui um efeito inibitório na atividade da $\mathrm{AChE}$ quando associado à indução de diabetes com aloxano no cerebelo e córtex cerebral, e um aumento na atividade dessa enzima por si só, no estriado. Isto indica que essa planta em associação ou sozinha pode induzir alterações na funcionalidade do sistema colinérgico nas estruturas estudadas.

\section{FONTES DE AQUISIÇÃO}

a- SUPRA LAB, Alisul Ind. Alimentos Ltda, São Leopoldo, RS. b- ALLOXAN MOHYDRATE, Sigma-Aldrich Inc, St Louis, MO.

\section{REFERÊNCIAS BIBLIOGRÁFICAS}

BIESSELS, G.J.; VAN DER HEIDE, L.P.; KAMAL, A. Ageing and diabetes: implications for brain function. European Journal of Pharmacology, v.441, n.1-2, p.1-14, 2002 .

BRAGANÇA, L.A.R. Aspectos gerais no preparo e no controle de qualidade de plantas e fitoterápicos hipoglicemiantes. IN: 
SIXEL, P.J. Plantas medicinais antidiabéticas: uma abordagem multidisciplinar. Rio de Janeiro : Universidade Federal Fluminense, 1996. Cap.5, p.105-122.

CHAKRABORTY, D.; MAHAPATRA, P.K.; CHAUDHURI, A. A neuropsychopharmacological study of Syzygium cumini. Planta Medica, n.9, p.139-143, 1986.

ELLMAN, G.C. et al. A new and rapid colorimetric determination of acetylcholinesterase activity. Biochemical Pharmacology, v. 21, n. 19, p.88-95, 1961

GROVER, J.K.; VATS, V.; RATHI, S.S. Anti-hyperglicemic effect of Eugenia jambolana and Tinospora cordifolia in experimental diabetes and their key metabolic enzymes involved in carbohydrate metabolism. Journal of Ethnopharmacology, v.73, p.461-470, 2000 .

KHANDKAR, M.A. et al. Alloxan-diabetes alters kinetic properties of membrane-bound form, but not of the soluble form, of acetylcholinesterase in rat brain. Biochemical Journal, v.307, p.647-649, 1995.

LAKHMAN, S.S.; KAUR, G. Effect of alloxan-induced diabetes on acetylcholinesterase activity from discrete areas of rat brain. Neurochemistry International, v.24, p.159-163, 1994.

LIMA, T.C.M. et al. Behavioural effects of crude and semipurified extracts of Syzygium cuminii Linn. Skeels. Phytotherapy Research, v.12, p.488-493, 1998.

MARCEL, V. et al. Two invertebrate acetitylcholinesterases show activation followed by inhibition with substrate concentration. Biochemistry Journal, v.329, p.329-334, 1998.

MILATOVIC, D.; DETTBARN, W-D. Modification of acetiylcholinesterase during adaptation to chronic subcute paraoxon application in rat. Toxicology and Applied Pharmacology, v.136, p.20-28, 1996.

MÜLLER, T.C. et al. Antidepressants inhibit human acetylcholinesterase and butyrylcholinesterase activity. Biochimica et Biophysica Acta, v.1587, p.92-98, 2002.

PRINCE, P.S.M.; MENON, V.P.; PARI, L. Hypoglycaemic activity of Syzygium cumini seeds: effect on lipid peroxidation in alloxan diabetic rats. Journal of Ethnopharmacology, v.61, p.1-7, 1998.

ROCHA, J.B.T.; EMANUELLI, T.; PEREIRA, M.E. Effects of early undernutrition on kinetic parameters of brain acetylcholinesterase from adult rats. Acta Neurobiological Experimentalis, n.53, p.431-437, 1993.

SCREMIN, O.U. et al. Cholinesterase inhibition improves blood flow in the ischemic cerebral cortex. Brain Research Bulletin, v.42, p.59-70, 1997.

SOARES, J.C.M.; COSTA, S.T.; CECIM, M. Níveis glicêmicos e de colesterol em ratos com diabetes mellitus aloxano induzido, tratados com infusão de Bauhinia candicans ou Syzygium jambolanum. Ciência Rural, v.30, p.113-118, 2000.

TAYLOR, P.; BROWN, J.H. Basic neurochemistry: molecular, cellular and medical aspects. 5.ed. New York : Raven, 1994, p.213-260. 